\title{
CCR6 mediates the intracellular HIV inhibitory activity of human beta-defensin 2 Lingling Sun ${ }^{1}$, Leon DeMasi ${ }^{2}$, Mark Lafferty ${ }^{2}$, Marco Goicochea ${ }^{2}$, Wuyuan Lu ${ }^{1}$ and Alfredo Garzino-Demo*1,2
}

\author{
Address: ${ }^{1}$ Institute of Human Virology, University of Maryland Biotechology Institute, Baltimore, Maryland, USA and ${ }^{2}$ University of Maryland, \\ Baltimore, Maryland, USA \\ * Corresponding author
}

\author{
from 2006 International Meeting of The Institute of Human Virology \\ Baltimore, USA. 17-2I November, 2006 \\ Published: 21 December 2006 \\ Retrovirology 2006, 3(Suppl I):S77 doi:I0.1I86/1742-4690-3-SI-S77
}

(c) 2006 Sun et al; licensee BioMed Central Ltd.

Recently, we and others have characterized the HIV-suppressive activity of human $\beta$-defensins (hBD) $1-3$. Our mechanistic studies indicate that hBD2 inhibits HIV through two distinct mechanisms. The first mechanism results in lower HIV infectivity. The second mechanism is intracellular, since it is observed even delaying treatment up to 2 hours after infection, which is not consistent with a mode of inhibition based on entry-fusion events. We hypothesized that this second antiviral mechanism is due to changes in the intracellular environment, possibly mediated by a cellular receptor. Treatment of cells in infectivity assays with an antibody specific for CCR6, a chemokine receptor that also functions as a receptor for hBD2, abrogated HIV inhibition by this defensin. Further, treatment of HIV-1 infectivity assays with MIP- $3 \alpha$, the cognate ligand for CCR6, resulted in inhibition of infection. Finally, single-cycle HIV infection is inhibited in cells that express CCR6 following transfection with an expression construct, but not in their CCR6low counterpart. Experiments performed using HIV pseudotyped with AMLV envelope showed that inhibition by hBD2 of HIV replication is sensitive to treatment with the $\mathrm{Gi}$ protein inhibitor pertussis toxin.CCR6 is an important receptor in the context of mucosal immunity, as it is expressed in CD4+CD45+CCR5+ lymphocytes, which are highly susceptible to HIV infection, and on dendritic cells, which are thought to play a crucial role in early events in HIV transmission. Therefore, an HIV suppressive mechanism target- ing CCR6 is likely to play an important role in mucosal immunity to HIV. 